\title{
A DYNAMIC RISK ASSESSMENT MODELING BASED ON FUZZY ANP FOR SAFETY MANAGEMENT SYSTEMS
}

\author{
Masoud REZAEI ${ }^{1}$, Naimeh BORJALILU ${ }^{*}$ \\ ${ }^{1}$ Civil and Environmental Engineering, Concordia University, Montreal, Canada \\ ${ }^{2}$ IranAir Co., Tehran, Iran
}

Received 18 May 2018; accepted 23 September 2018

\begin{abstract}
Risk assessment in large organizations with extensive operational domains has been a challenging issue. Employing an efficient method along with realistic pair comparisons, applying subjective inferences of organization experts, and purging the intrinsic ambiguity of inferences, are not reflected in current airlines' safety management. Traditional twodimensional risk assessment for risk management of safety hazards, however, is no longer sufficient to comply with this complexity. A new model for risk management and a novel formula for risk index calculation, based on a fuzzy approach, are presented in this study.

In this new model, unlike in the traditional approach, the latent aftermath of safety reports, especially those which affect the continuity of the business, is also taken into account. In this model, along with the definition of a new structure for risk management, risk analysis should be restructured. To that end, a two-dimensional classic risk formula was replaced with three-dimensional (nonlinear) exponential ones, considering "the impact on the business" as a source of risk and hazard. For measuring the safety risk using the Fuzzy hierarchical evaluation method, considering experts' opinions, three criteria in four different operational fields were developed.

This method employs a Fuzzy ANP to help quantify judgments, make qualitative judgments in the traditional method, and weigh the priority of elements contributing to risk. Also, it provides a tool for top-level as well as expert level management to monitor safety more precisely, monitor the safety level within their departments or organizations, set quantitative safety goals and provide feedback for improvement as well as find the most critical areas with the least cost. In this study, an airline has been selected as a case study for the risk assessment of reports based on the new model.
\end{abstract}

Keywords: fuzzy, ANP, safety risk assessment, impact on business, aviation.

\section{Introduction}

Evaluation of airlines based on service quality criteria can help to improve the working processes of airlines and can give guidance to travel agencies to provide better choices for passengers and tourists (Ghorabaee, Amiri, Zavadskas, Turskis, \& Antucheviciene, 2017). The next generation of Air Transport Management (ATM) requires performancebased safety management that is fully integrated into seamless operational management (Kontogiannis, Malakis, \& Mcdonald, 2017).

Among businesses, there is an incentive to invest in management oversight, as a series of accidents could hurt the reputation and profitability of the industry. An adverse environmental or safety record within the industry may affect market access, which could otherwise be denied to the industry. Conversely, regulators may reward firms who participate in self-audit and disclosure programs, such as reducing the number of future inspections or increasing access. These incentives help to provide a strong market incentive to businesses, which in turn promotes better compliance (Oldhama, Stantonb, Bilderbecka, \& Spinettoa, 2017).

Over the last ten years, the world's commercial aviation system industry has improved its overall safety performance by $54 \%$, with an accident rate in 2016 of 1.61 accidents per million sectors, compared to 3.53 in 2007. The 2016 accident rate, which includes all accidents, was $10 \%$ lower than in 2015. However, this overall positive performance was counter-balanced by an increase in the number of fatalities, fatal accidents and hull losses versus the previous year. With a total number of fatalities at 268 , 2016 represented an increase of $97 \%$ over 2015 . Of the ten

*Corresponding author. E-mail: borjalilu@gmail.com 
fatal accidents in 2016 (six more than in 2015), six were on cargo flights. On the positive side, 2016 is still below the previous five-year average of 371 fatalities per year (International Air Transport Association [IATA], 2017).

Left alone and poorly managed, most organizations will become less safe. Management neglect, worker apathy, and an absence of analysis, will all eventually create a less-safe operation. On the other side of the equation, a successful Safety Management System (SMS) can produce very positive safety outcomes. In this sense, a safety management system (SMS) is clearly promising for the business of air transportation. The introduction of a safety management system (SMS) in the real operational environment has become a key factor in a proactive view of flight safety.

In 2012, the European Aviation Safety Agency (EASA) released the first Implementing Rules (IRs) addressing safety management requirements for Authorities, Aircrews and Air Operators EC (2012a, 2012b). Indeed, EASA introduced an integrated approach to the SMS implementation process since the SMS should be fully integrated in the organisation's existing management system, and safety management should include every facet of management that may impact aviation safety (Panagopoulos, Atkin, \& Sikora, 2017).

The Federal Aviation Administration (FAA) implements a safety management system (SMS) that provides the nation with the safest airspace in the world. The SMS is built on four pillars: safety policy, safety risk management (SRM), safety assurance (SA), and safety promotion (Wise, 2016).

IATA is another active participant of safety establishment in aviation that implements the SMS and categorizes it into safety performance monitoring, analysis and dissemination of information, and safety promotion and facilitation. Risk assessment and management are the core of all these areas and provide grounds for the development of all processes by using and analyzing the gathered data.

The International Civil Aviation Organization (ICAO) has stablished a framework for aviation safety, described in Annex 19 as a safety management system (SMS). This annex was a response to requests raised globally for devoting a specific annex to safety for a full coverage of safety-related problems. The main purpose was to address safety risks and put the stress on the concept of overall safety performance. All parts of the SMS, namely Safety Policy and Objectives, Risk Management, Safety Assurance, and Safety Promotion, are directly or indirectly dependent on risk assessment (Wise, 2016). Based on ICAO's annex 19, operators shall be responsible for establishing an SMS, which is accepted and overseen by their State.

ICAO itself recognized the framework for the SMS, which includes the following components and elements:

1. Safety policy and objectives.

2. Safety risk management.

3. Safety assurance.

4. Safety promotion.
Within the context of aviation, safety is defined as "the state in which the possibility of harm to persons or of property damage is reduced to, and maintained at or below, an acceptable level through a continuing process of hazard identification and safety risk management". In the context of an SMS, hazard identification is a prerequisite to the safety risk management process. Any incorrect differentiation between hazards and safety risks can be a source of confusion. A clear understanding of hazards and their related consequences is essential to the implementation of sound safety risk management (DOC 9859-AN/474 (International Civil Aviation Organization [ICAO], 2013a)).

A safety risk is the projected likelihood and severity assigned to the consequence of a predicted or existing hazard or situation. While the outcome may be an accident, an "intermediate unsafe event/consequence" may be identified as "the most credible outcome". The provision for the identification of such layered consequences is usually associated with more sophisticated risk mitigation software (ICAO, 2013b). There are few possible risks that can occur during a production process. Risks can lead to a total failure in reaching the goal of production, or can even lead to a hazard (Noriyati, Rozaaq, Musyafa, \& Soepriyanto, 2015).

Principles and methods have been developed for how to conceptualize, assess and manage risk. These principles and methods still largely represent the foundation of this field today, but many advances have been made, linked to both the theoretical platform and practical models and procedures. The risk field has two main tasks:

- to use risk assessments and risk management to study and treat the risk of specific activities, for example, the operation of an offshore installation or an investment;

- to perform generic risk research and development, related to concepts, theories, frameworks, approaches, principles, methods, and models, in order to understand, assess, characterize, communicate, and (in a wide sense) manage/govern risk (Aven, 2016).

There are many factors on different levels which affect aircraft flight safety evaluations, as there are many uncertainties and significant mutual interferences between the levels, so it is difficult to attain accurate measurements (Deng, Wang, \& Liang, 2017). Major outputs are utilized to identify and implement measures intended for maintaining risk at an accepted level. Risk assessment can be materialized as a process originating from hazards, considering sufficiency of all existent controls. There are various definitions for risk. It is defined as "the probability of occurrence of a hazardous event in a given period". It may be considered as the possibility that an individual or group get impaired through the aftermath of specific actions in a random manner. Risk can also be linked to a statistically expected value of loss (i.e. the statistical likelihood of a randomly exposed individual being affected by some hazardous event) (Janic, 2000). 
Risk assessment and modeling to deliver reliable results have long been the subject issue for researchers. Although aviation is considered as the safest among other types of transportation means, it is also regarded as the most expensive. Despite the fact that the rate of incidents and accidents in aviation is low compared to railway or road transportation, the cost of these occurrences is much higher. The term of cost, here, includes immediate and belated costs. Immediate costs are the money which is spent to recover or renew the aircraft, vehicles, equipment, humans, and training, and make up for penalties. Belated costs are the costs which are not counted directly or considered when there is no immediate loss, damage or fatalities in the safety reports received. Nevertheless, they can drastically incur cost to operators. Delays in flight, abortive take-off, hard landing causing fear in passengers, cancelled flights that cause disruption to normal operations, and chain delays in other flights, are all examples of belated costs. These events have a negative impact on the operator's reputation over time and result in a reduction of revenue and loss of the market.

Accidents are the objects of investigations for hazard identification via risk assessment. To evaluate the cost incurred by accidents and incidents, a two-dimensional linear formula was derived for assessing the risk. However, a two-dimensional matrix in risk assessment mostly undermines the flexibility of the model and future improvements. Impartial risk assessment without or with less human judgement, bias, and prejudice must be carried out to ensure the appropriateness of hazard identification and effectiveness of corrective actions for risky items. With a two-dimensional matrix for risk assessment, it is difficult to preserve impartiality due to the qualitative assessment of risks. Therefore, a more flexible and purposeful risk assessment is necessary to include the most important safety risks. The likelihood of failures at lower levels of employees and a higher level of managerial structure are interconnected.

New methods of risk assessment require a modular approach that would simplify the process. Simplification of assessment means developing models in which general users can tackle assessment easily, while at the same time its accuracy in not diminished. The input and output variables and operational relationship are vital to evaluate the level of management's impact on risk.

Typically, the complexity of the problems is reflected more or less in the models. However, the value of the models is also defined based on these characteristics. In large organizations, risks are complicated and the risk assessment model should be simple enough to cover all of the technical and human parameters, while covering management level actions or decisions in the evaluation of the risks. Today's models should be able to predict the systems' response in both normal and emergency states via performance indicators. They should take into account other types of dependencies like feedback and feedforward data, or information transfer for data analysis.
Since risk assessment is complex and ambiguous, using qualitative terms is unavoidable. Additionally, the perceptions on likelihood and the impact of risk factors on respondents are typically subjective and uncertain. The fuzzy set theory can deal with the problems relating to ambiguous, subjective, and imprecise judgments. This theory also allows mathematical operators to be applied to the fuzzy domain and can quantify the linguistic facet of the available data and preferences for individual or group decision-making. Thus, the fuzzy set theory is considered to be an appropriate tool for risk assessment.

To summarize, conventional approaches are incapable of providing a comprehensive risk assessment in different sections and a quantitative risk variable to represent the overall risk status of the system, integrating different databases, and considering economic consequences, such as labor hours, materials and facilities, and credit losses, because of inappropriate risk assessments. To enhance conventional risk assessment techniques by compensating for the above-mentioned deficiencies in these approaches, a new risk modeling and a novel formula for the risk index are proposed in the current paper, providing a priority of corrective actions, improving the safety level of the organization, and considering the economic impacts.

Multi-Criteria Decision-Making (MADM) is used for finding the best choice amongst all possible options for decision making. These models are formulated in the form of a decision matrix. One of the techniques of multi-criteria decision-making is the ANP, which is suitable for most complicated problems. The ANP allows modeling if internal relations exist between the different levels of decisions and criteria. The ANP was developed to generate priorities for decisions without making assumptions about a unidirectional hierarchy relationship between decision levels. One of the ANP's drawbacks is the lack of uncertainty and lack of complete reflection of human thinking in modeling. This kind of uncertainty in priorities can justify the Fuzzy method. In other words, Fuzzy models are more compatible with linguistic and often ambiguous explanations to carry out long term predictions and to make decisions in the real world. In Fuzzy literature, the rate produced by the decision taker is a fuzzy number originating from the membership function. This function specifies the membership degree of each member. Therefore, an exact description of each decision-making process is presented via merging fuzzy concepts with the ANP (FANP).

This article aims to propose a new approach for appraising reports one by one in each section, integrating different criteria mathematically into one index in order to derivate an overall safety index of the organization for top management. Using multi-attribute decision-making in conjunction with the implementation of the fuzzy numbers structure can be regarded as an efficacious method for determining the significance of each criterion and option. Consequently, these criteria will collectively form a numerical safety report index with the aid of the FANP (to implement realistic comparisons as well as precisely 
draw inferences from each filed expert). The novelty of this paper is the introduction of a three dimensional exponential (nonlinear) formula for the calculation of risk index instead of the two dimensional linear formula, which enables the definition of safety indicators at low, medium and top-level management of big organizations. It facilitates the definition and monitoring of the safety goals by the management to find the most critical areas with the least cost.

The methodology used in this article is 3D risk assessment of safety-related reports via a Fuzzy ANP (Fuzzy Analytical Network Process) based method which consists of three distinct criteria indicating severity of occurrence, reliability (frequency of occurrence), and the impact on business and also options which indicate the safety reports of several sectors in an airline.

\section{Literature review}

\subsection{Research theoretical framework}

The aviation system is complex and dynamic, influenced by human, machine, environment, and many other factors. The study of aviation system safety engineering is one of the branches of aviation safety management (Xueyan, Mingliang, \& Mingang, 2012). Recently, the global aviation industry has started to promote the Safety Management System (SMS) thus considering the enhancement of safety culture an essential issue (Tsay, Kuo, Chao, Drury, \& Hsiao, 2014). Safety management is a multidisciplinary area, which draws heavily from fields as diverse as economics, engineering, industrial relations, law, management, occupational hygiene, occupational medicine, psychology, and sociology (Pillay, 2015). Managing safety has become increasingly more important in aviation and other high-reliability and safety-critical systems. Safety culture will play a more critical role in improving safety and, as the International Civil Aviation Organization has pointed out, "an effective way to promote a safe operation is to ensure that an operator has developed a positive safety culture" (Wang \& Sun, 2012).

The ever-increasing complexity of operation, together with the need to obtain efficient processes with limited costs, has led companies to develop analytical and computational tools for risk assessment. Safety management and its integral part, risk management, have walked a long way to their current destination. Risk management defined as "a set of activities for the hazard identification process, verification and risk assessment and, eventually, the execution of a control program in order to decrease or eliminate the identified hazard risks, probable outcomes, and the documentation of final decisions" (DOC 9859AN/474 (ICAO, 2013a)).

Alongside these issues and adopted recommendations through the SMS, many researchers have worked on safety management and risk assessment. The characteristics of accidents were firstly dealt with through the introduction of the "Domino Theory" in the early 1930s. In this model, different elements in the chain of events were connected to lead to an injury or accident. Among them were societal culture, human error, and the operator's background (Griffin, 2010). The focus of this model originated from the military and industrial domains in which the goal is to reduce the number of injuries and financial losses generated by unsafe acts. As one of the first attempts, ICAO tackled safety with the "Machol Reich" model in the late 1960s (Netjasov \& Janić, 2008). Also, in this new model, the number of accidents mattered more than the prediction of accidents through risk assessment and management. This model was suffering from ignoring human factors and organizational culture as the major parameters in accidents and incidents. This deficiency was supposed to be covered in new models introduced after the 70s.

As the first step to integrate human-related elements with equipment-originated factors, management-related factors were considered by Weaver (1971). This approach in modeling was continued by (Bird \& Loftus, 1976). This stream was taken and followed by (Adams, 1976) as he introduced organizational error as an element. However, the mostly accepted and widely used model was the Swiss cheese model introduced by James Reason (1975) (Reason, 1998). It relied on the error discussion techniques and barriers of Johnson (Johnson, 1975). The logic behind this is to compare an accident to an arrow passing through the rotating cheese slices when the holes (representing the deficiencies, flaws, human errors, design shortcomings, etc.) are aligned. In the new millennia, new requirements and rapidly changing environment introduced some novel threats, hazards and risks associated with new changes. Therefore, the Swiss cheese model had to be updated or expanded to consider these new requirements.

Perrow (1999) introduced a theory in which a "normal accident" describes the complicated nature of relations between the systems leading to an accident (Perrow, 1999). It was then followed by Reason. Reason carried out an intensive study of organizational factors and the structures playing a role in accidents and incidents (1998). Rijpma proposed the high reliable organization term (HRO) to describe the safety management system in a complex organization (Sikora, 2015). Resilience was defined by Hollnagel, Woods, and Leveson (2007) after organizational factors contributing to system safety were thoroughly applied for safety and risk modeling in a research study (2007). Analysis of organizational structure and its response to developing safety issues was feasible via this approach (ICAO, 2013b; Cacciabue, Cassani, Licata, Oddone, \& Ottomaniello, 2015). Rasmussen's (1997) results were strong enough to persuade Leveson to conclude that the identification of the interrelation between systems inside an organization is crucial for accident prevention.

Paying attention to the operator's activities, equipment failures or design flaws only is not sufficient to fully consider and entirely cover the prevention activities and tactics for future accidents. Risk modelling should address more than the accident mechanisms for more comprehensive investigation practices (Sikora, 2015). 
According to Stoop, users need various data to identify systemic hazards. Users need data for the analysis while management and regulators need indicators and means for monitoring safety of the system operation (Stoop \& Dekker, 2010).

Based on SMS theories, Shengguo and Xianfeng derived criteria in three different categories: human factors, facility and equipment factors, and environment factors. For each of them, related indices have been assigned and, for the integration and measurement of indices, a mathematical model has been introduced to ascertain how and how much each index contributes and influences the whole system (Xianfeng \& Shengguo, 2012). Xueyan et al. (2012) presented a qualitative model to evaluate the safety risk of flight-oriented operations so that, by functional approximation, it becomes possible to explore dangerous and safe regions and measure the safety risks extracted from flight data via a sample investigation.

Another study in the field of risk assessment of maintenance resulted in a checklist for the evaluation of all items from all fields affecting risk assessment (Lind, Nenonen, \& Kivistö-Rahnasto, 2008). With regards to an airport and its relevant operations, Zhao, L. Liu, and F. Liu (2008), conducted another study using the FAHP method for risk assessment.

The ever-increasing complexity of production systems, together with the need to obtain efficient processes with limited costs, has led companies to develop custom tools for process control and management. Even for risk assessment, traditional models are often overcome by methods that are best suited to specific needs, which classify risks and identify corrective actions that allow the best risk reduction at the lowest cost (Di Bona, Silvestri, \& De Felice, 2016). Risk analysis is associated with decision making. The traditional approach to risk assessment entails a qualitative comparison of phenomena. As such, human bias, judgement, and culture are highly mixed in judgements. In this context, when there are no rigid quantitative values, risk assessment needs decision-making. Various techniques for decision-making have been justified in this field. One of them is the multi decision-making technique.

Multi decision-making approaches are typical while dealing with different measures collectively. At present, for carrying out performance evaluation, hierarchical analysis is at the top of the agenda, when considering measures. However, the measures are not always independent and have mutual interactions. Therefore, Saaty introduced the Fuzzy Analytical Network Process, which is a development of the hierarchical analytical process to obtain a package of proper weights (Tuzkaya \& Önüt, 2008). Another study using ANP for risk assessment, performed by RamKumar, Schoenherr, and Jenamani, resulted in identifying eprocurement risk factors through a strength, weaknesses, opportunities, threats analysis, grounded in transaction cost economics (TCE), and proposing a risk assessment framework based on the opinions of a group of experts (Ramkumar, Schoenherr, \& Jenamani, 2016). In another study, a modified ANP and fuzzy inference system based approach was used for risk assessment of in-house and third party e-procurement systems (Ramkumar, 2016).

In classical management, rational and systematic methods for management problem analysis based on precise and logical data have no role to play. Classical management science is used in a Fuzzy environment. Fuzzy management science with embedded flexibility considers factors such as experience, knowledge, and judgment in its medialization and presents practical answers (Yuksel \& Dagdeviren, 2011).

The formalisation of Fuzzy sets started in the 1960s with the works of Zadeh in Fuzzy sets and Dempster in belief functions, set functions, which generalize additive probability measures. Belief functions offer a non-Bayesian method for quantifying subjective evaluations by using probability. Fuzzy Logic can handle problems with imprecise data and give results that are more accurate. The fuzzy preference programming (FPP) method is mainly used to derive priority vectors from a set of comparison judgments or interval comparisons (Lin, 2009). In Fuzzy Logic, the logic operations method is based on manyvalued logic rather than binary logic or two-valued logic. Fuzzy Logic deals with truth-values between zero and one, and these values are considered as intensity or degrees of truth. Zadeh introduced the fuzzy set theory to deal with the uncertainty due to imprecision and vagueness. A major contribution of fuzzy set theory is its capability of representing vague data (Maseleno, Hasan, Tuah, \& Tabbu, 2015; Yüksel \& Dagdeviren, 2010).

\subsection{Fuzzy multi criteria analysis}

In recent years, many techniques to solve Multi-Criteria Decision-Making (MCDM) problems have been proposed (Wichapa \& Khokhajaikiat, 2017). In MCDM, instead of using one optimization measure, a number of evaluation criteria are used. These are divided into two main groups MODM (Multi Objective Decision Making) and MADM (Multi Attribute Decision Making). In general, MODM is preferable for design and MADM for the sake of the best choice. The main distinctive difference between the MADM and MODM is that MADM is defined in a continuous decision-making environment and a discrete decision-making environment. The above-mentioned methods in connection with Fuzzy logic are now studied as Fuzzy Multi Objective Decision Making (FMODM) and Fuzzy Multi Objective Decision Making (FMADM).

\subsubsection{Fuzzy Multi Attributed Decision Making (FMADM)}

As stated before, MADM is used to determine the best choice amongst all possible options. These models are formulated in the form of a decision matrix. In this matrix, $A_{i}$ denotes the $i^{\text {th }}$ option, $x_{i}$ denotes the $j^{\text {th }}$ indicator, $r_{i j}$ denotes the $j^{\text {th }}$ indicator value for the $i^{\text {th }}$ option. MADM methods involve 2 steps: consensus of viewpoints regarding indicators, and options and options order on the basis of collective viewpoints. 
In absolute MADM models, it is assumed that the ultimate viewpoint about one option is stated in a quantitative manner via a number. However, in real circumstances, the possibility of not having this assumption may lead to the incapability of taking the advantage of absolute numbers for expressing the importance of the indicators or the value of the options with respect to various parameters. In such circumstances, the FMADM is preferable. YAGER, Bonison, FANP, and FAHP are methods that are founded on the FMADM models (Zadeh, 1965).

\subsubsection{FANP method}

One of the primary techniques among multi-criteria decision-making techniques is the AHP, which is suitable for most complicated problems. Saaty founded ANP and proposed it as a generalization of the AHP. While the AHP provides a context for hierarchical structures with single direction relations, the ANP allows modeling if internal relations between different levels of decisions and criteria exist. The ANP was developed to generate priorities for decisions without making assumptions about a unidirectional hierarchy relationship between decision levels (Dargi, Anjomshoae, Galankashi, Memari, \& Tap, 2014).

One of the ANP's drawbacks is the lack of uncertainty and lack of complete reflection of human thinking in modeling. This kind of uncertainty in priorities can justify the Fuzzy method. In other words, using Fuzzy models has more compatibility with linguistic and often ambiguous explanations to carry out long term predictions and to make decisions in the real world. In Fuzzy literature, the rate produced by the decision taker is a fuzzy number originating from the membership function. This function specifies the membership degree of each member. Therefore, an exact description of each decision-making process is presented via merging fuzzy concepts with ANP. By presenting the Fuzzy collection theory, Zadeh interprets and justifies the uncertainty related to resulting ambiguity and conception as a kind of human thought simulation. Fuzzy ANP is the development of ANP. The Fuzzy numbers used in this method are triangular Fuzzy numbers and the Fuzzy scale utilized in this method is illustrated in Table 1.

These scales are used for pair comparison. Suppose two triangular fuzzy numbers:

$$
\begin{aligned}
& M_{1}=\left(L_{1}, m_{1}, U_{1}\right), M_{2}=\left(L_{2}, m_{2}, U_{2}\right) ; \\
& M_{1}+M_{2}=\left(L_{1}+L_{2}, m_{1}+m_{2}, U_{1}+U_{2}\right) \\
& M_{1}^{-1}=\left(\frac{1}{U_{1}}, \frac{1}{M_{1}}, \frac{1}{L_{1}}\right) \quad M_{2}^{-1}=\left(\frac{1}{U_{2}}, \frac{1}{M_{2}}, \frac{1}{L_{2}}\right) ; \\
& M_{1} \times M_{2}=\left(L_{1} L_{2}, m_{1} m_{2}, U_{1} U_{2}\right) .
\end{aligned}
$$

In the equations above, $L_{i}$ is the lower limit, $\mathrm{m}_{\mathrm{i}}$ is the average limit, and $U_{i}$ - the upper limit of a triangular number, known as optimistic, indifferent (average) and pessimistic limits of opinions. Figure 1 depicts two triangular fuzzy numbers on a coordinate axis (Zhao, Hwang, \& Gao, 2016).

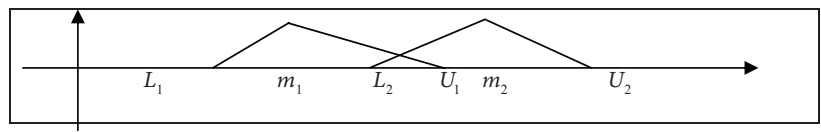

Figure 1. Two triangular fuzzy numbers $\left(M_{1}\right.$ and $\left.M_{2}\right)$ on a coordinate axis

Table 1. Triangular Fuzzy scale definitions

\begin{tabular}{|l|l|}
\hline \multicolumn{1}{|c|}{ Reciprocal scale } & \multicolumn{1}{c|}{ Linguistic scale } \\
\hline $\mathrm{A}=(1,1,1)$ & Just equal \\
\hline $\mathrm{B}=(2 / 3,1,3 / 2)$ & Equally important \\
\hline $\mathrm{C}=(3 / 2,2,5 / 2)$ & Strongly more important \\
\hline $\mathrm{D}=(5 / 2,3,7 / 2)$ & Very strongly more important \\
\hline $\mathrm{E}=7 / 2,4,9 / 2))$ & Absolutely more important \\
\hline
\end{tabular}

The steps of an FANP analysis can be described as follows (Lin, 2009).

Step 1. The value of the fuzzy synthetic extent with respect to the $i^{\text {th }}$ object is defined as:

$$
S_{i}=\sum_{j=1}^{m} M i j \times\left[\sum_{i=1}^{n} \sum_{j=1}^{m} M_{i j}\right]^{-1} .
$$

Step 2. The degree of possibility of $\mathrm{S} 2 \geq \mathrm{S} 1$ is defined as:

$$
\begin{aligned}
& \left.V=(S j\rangle S_{i}\right)=\operatorname{height}\left(S_{i} \cap S j\right)=U(d)= \\
& \left\{\begin{array}{cc}
1 & m_{j}>m_{i} \\
0 & L_{i} \geq U_{j} \\
\frac{l_{i}-u_{j}}{\left(m_{j}-u_{j}\right)-\left(m_{i}-l_{i}\right)} \text { otherwise }
\end{array}\right\} .
\end{aligned}
$$

Step 3. The degree of possibility for a convex Fuzzy number to be greater than $\mathrm{K}$ convex fuzzy numbers $\mathrm{M}_{\mathrm{i}}$ $(\mathrm{i}=1,2, \ldots \ldots \ldots, \mathrm{k})$ can be defined by:

$\left.V=(S\rangle S_{1}, S_{2}, \ldots . ., S_{k}\right)=V\left[(S\rangle S_{1}\right)$ and $\left.\left.\left.\ldots . . .(S\rangle S_{k}\right)\right]=\min V(S\rangle S_{i}\right)$ for $i=1,2, \ldots . . k$

$d^{\prime}\left(A_{i}\right)=\min \left(S \geq S_{i}\right) \quad$ for $\quad i=1,2,3, \ldots \ldots \ldots . .$.

Step 4. Via normalization, the normalized weight vectors are

$$
W^{\prime}=\left(d^{\prime}\left(A_{1}\right), d^{\prime}\left(A_{2}\right), \ldots \ldots \ldots \ldots \ldots . . . . d^{\prime}\left(A_{n}\right)\right)^{T},
$$

where $W$ is a non-fuzzy number:

$$
W=\left(\left(d\left(A_{1}^{\prime}\right), d^{\prime}\left(A_{2}\right)^{\prime}, \ldots \ldots \ldots \ldots \ldots . . . d^{\prime}\left(A_{n}\right)\right)^{T} .\right.
$$

\subsubsection{Gap analysis}

There are some gaps in the modeling of studies that were described above. These gaps were the motivation to develop a new model for risk assessment and management. 
Many of the shortcomings are found at the beginning of the risk assessment, namely during the calculation of the risk index.

One of the main concerns for the implementation of a classical risk management system in large organizations is the existence of a vast number of subsections and operational sectors with sophisticated duties. Conventional approaches are incapable of providing a comprehensive risk assessment in different sections, with a tangible quantitative indicator. They are also unable to empower safety management systems to monitor the risk of hazards, define trackable safety goals and prioritize corrective actions.

In two-dimensional risk, the parameters used for the assessment of the severity of financial or fatal losses and the probability of accidents/incidents are defined as follows:

Risk Index (RI) $=$ Probability ${ }^{\star}$ Severity,

where severity denotes the possible effects of an unsafe event or condition, taking into account the worst foreseeable situation, and probability is the likelihood of an unsafe event or condition that might occur.

Different classifications of risk levels can be assumed, based on three levels classified as low, medium, high, or five levels - very low, low, medium, high, very high (Figure 2). The two-dimensional approach has a number of deficiencies.

Firstly, in the traditional approach, which is illustrated in Figure 2, a number or a combination of a number and a letter are assigned to each report. This approach focuses on analyzing each report individually. Although there are advantages for this kind of assessment, such as simplicity, the disadvantages heavily outweigh the advantages. Consideration of each report without a comparison with other reports describing incidents or accidents largely lies in the experience and judgement of the expert. If the expert has no skill in the field, it may cause an overestimation or un- derestimation of the risk in the report. Secondly, without a comparison, the prioritizing of corrective actions and the resource allocation are a matter of difficulty.

Thirdly, the risk index derived for each report in the classical format is not flexible enough to easily indicate the safety trend of a section, department, or the whole organization. In fact, a multitude of reports with a risk index in digit-letter format, which is visible in Figure 2, like 5C or a non-normalized number, are yielded. They are regarded solely and could not be integrated in a logical manner to give an indicator for the section reports.

Moreover, in the assessment of risks, the safety policies declared by top-level management are not reflected. Based on their perspective of the probability or the judgment of severity, experts assign the risk index. However, organizations have a mission and vision of operation that meet their requirements. For example, for a military organization, the continuity of work is more important than cost, financial loss, or discontinuity of the operation, whereas in a civil aviation company, like an airline, costs are more important than continuity of operations. These policies shall be considered in the risk analysis in line with the organization's mission. In the traditional risk assessment approach, there is no possibility for to include managerial views or organizational policies.

All of these shortcomings are covered in the new model and formula proposed in this article. Because of these drawbacks, researchers are unable to design a Safety Management System that covers all sections and activities of large organizations. As an example, in airlines, the risks for on-board status reports have been identified and categorized. However, the safety reports of on ground status of an aircraft or ground services are mostly ignored or rarely considered. This is a big deficiency of the traditional risk assessment approach. In addition, researchers were primarily unable to propose a safety management system

\begin{tabular}{|c|c|c|c|c|c|}
\hline \multirow[t]{2}{*}{ Risk probability } & \multicolumn{5}{|c|}{ Risk severity } \\
\hline & Catastrophic & Hazardous & Major & Minor & Negligible \\
\hline Frequent & $5 \mathrm{~A}$ & $5 B$ & $5 \mathrm{C}$ & $5 \mathrm{D}$ & $5 \mathrm{E}$ \\
\hline Occasional & $4 \mathrm{~A}$ & $4 \mathrm{~B}$ & $4 \mathrm{C}$ & $4 \mathrm{D}$ & $4 \mathrm{E}$ \\
\hline Remote & $3 \mathrm{~A}$ & $3 \mathrm{~B}$ & $3 \mathrm{C}$ & $3 \mathrm{D}$ & $3 \mathrm{E}$ \\
\hline Improbable & $2 \mathrm{~A}$ & $2 \mathrm{~B}$ & $2 \mathrm{C}$ & $2 \mathrm{D}$ & $2 \mathrm{E}$ \\
\hline $\begin{array}{l}\text { Extremely } \\
\text { improbable }\end{array}$ & $1 \mathrm{~A}$ & 1B & $1 \mathrm{C}$ & $1 \mathrm{D}$ & $1 \mathrm{E}$ \\
\hline \multicolumn{3}{|c|}{ Risk management } & \multicolumn{2}{|c|}{ Assessment risk index } & Suggested criteria \\
\hline & & & \multicolumn{2}{|c|}{$\begin{array}{l}5 \mathrm{~A}, 5 \mathrm{~B}, 5 \mathrm{C}, 4 \mathrm{~A} \\
4 \mathrm{~B}, 3 \mathrm{~A}\end{array}$} & $\begin{array}{l}\text { Unacceptable under the } \\
\text { existing circumstances }\end{array}$ \\
\hline & Tolerable & & \multicolumn{2}{|c|}{$\begin{array}{c}5 \mathrm{D}, 5 \mathrm{E}, 4 \mathrm{C}, 4 \mathrm{D}, 4 \mathrm{E} \\
3 \mathrm{~B}, 3 \mathrm{C}, 3 \mathrm{D}, 2 \mathrm{~A} \\
2 \mathrm{~B}, 2 \mathrm{C}\end{array}$} & $\begin{array}{c}\text { Acceptable based on risk mitigation. It might require } \\
\text { management decision }\end{array}$ \\
\hline & Acceptable & $\boldsymbol{\nabla}$ & \multicolumn{2}{|c|}{$\begin{array}{l}3 \mathrm{E}, 2 \mathrm{D}, 2 \mathrm{E}, 1 \mathrm{~A}, 1 \mathrm{~B} \\
1 \mathrm{C}, 1 \mathrm{D}, 1 \mathrm{E}\end{array}$} & Acceptable \\
\hline
\end{tabular}

Figure 2. 2D risk matrix including three risk levels 
capable of defining a quantitative risk index which could be reliable for investigations and monitoring for all departments and the whole organization.

Therefore, in this paper, along with a review and classification of the proposed criteria, the priority of parameters influencing the mathematical model is introduced, and, finally, the model and its implementation in a case study are presented.

\section{Methodology}

One of the main concerns about the implementation of a comprehensive risk management system for aviation and airlines in particular is the existence of a large number of subsections, sections, and operational divisions with sophisticated duties. As a result, the system performs inefficiently due to many input variables. Therefore, the risk management system is based on the extraction of apparent and hidden risk threats from the safety reports, so the decision-making process is to be based on proactive or reactive approaches.

To implement a comprehensive safety management system, the important steps are to provide a continuous monitoring and regular assessment of the achieved safety level as well as to improve the establishment of an overall level of safety in the data/report gathering system continuously. The possibility to have an overall quantitative safety indicator for the operations of the whole airline, while having access to the situation of each division separately, assists the board of directors in realizing the safety status of the organization, identifying the weaknesses, suggesting applicable corrective actions, and monitoring the impact of the decisions continuously. In the majority of cases, only the reports related to issues on-board aircraft and operations performed on the fleet on the ground are considered.

The reports are made from the moment that the flight license is issued for the plane up to the last moment of operation. This category includes all phases of a flight, unexpected events (those that have led to incidents and those that have not), human factors, technical objections, and weather conditions. However, in the new methodology, all the reports from ground support services, such as accidents in hangars, unsafe maintenance, FOD (Foreign object damage) on ramps, unsafe maneuver of vehicles in the safety zone around the aircraft, could be included in the calculation of the overall risk index of the organization. The sections included are engineering and maintenance, airport services, control tower, ramp handling, etc.

\subsection{Model development}

Each organization needs a safety indicator to assess its success or failures in the achievement of goals. Safety goals are usually defined in general terms or using qualitative expressions, which cannot be tracked simply and effectively. An overall safety indicator empowers the top-level management to monitor the safety of an organization and distinguish which zones or areas are responsible for the deterioration of safety. Based on safety monitoring results obtained using the overall safety indicator, feedback could be given to lower level divisions by issuing a new safety policy or certain allocation of resources.

The mission and vision of organizations are neither similar, nor fixed, and may change over time. For example, the mission of a military aviation company is different from that of a civil aviation organization; subsequently, the priority of a mission affects their perception and acceptance of hazards and risks. Moreover, the mission of a company may change when a domestic small air service company wants to play a greater role in the national or international domain. If there are no competition and delays in the domestic market, flight cancellation has either no or little impact on the revenue. However, internationally, the discontinuity of work has an impact on business and plays a role in the assessment of risks and identification of hazards.

Unlike in the classical approach, risk assessment is exponential rather than linear. The advantages of this new form of formula are expressing the priority of importance of the elements contributing to the risk, from the management viewpoint. In simple words, they show for which item, according to severity, probability and impact on business, it is more important or interesting for the company to allocate the resources or prioritize the actions needed. The strength of the new concept is providing the possibility to reflect the mission of operation in its risk management system to each organization. In addition, whenever the mission of an organization changes, new safety policies and priorities can be applied in risk assessment by changing the powers in the formula, something that was missing in the linear two-dimensional formula in classic risk assessment.

The new model also takes the FUZZY approach, because it makes pair comparisons of all reports, something that is not carried out in two-dimensional analysis. Using this approach, two goals are simultaneously achieved. Firstly, prioritization takes place when making the comparisons, which can then be completed by management prioritization in defining the powers. Meanwhile, the normalization of the risk elements, namely severity, probability, and impact on business is performed. One of the main problems associated with traditional risk assessment is the fact that the risk index and its contributing elements are not normalized. A risk index which has not been normalized may drastically affect the risks of reports and cause an error in corrective actions. The results of this approach are normalized digit numbers, which can show more precisely which risks need more attention. Based on their order, the type of action (immediate, normal and no actions) is defined. During the next phase, these risk numbers for each section can be summed up and divided by the number of reports received to deliver the total risk number of the sections. By repeating this for each department or section, a normalized risk index for all departments is yielded. 
At this level, each department can depict its total risk index on the axis of time and monitor its safety trend or define the safety goal by specifying a threshold for the safety index to be reached. For example, if the safety risk of a flight operation is 2.1 for the month of January, flight operation managers can define the department's safety goal at 2 , to be reached within the next 6 months. Therefore, defining the safety goal by monitoring the safety index is achievable in this method of risk assessment. In addition, top-level managers or chief executives of an organization are able to monitor the safety indexes of all departments and identify the most critical department from the safety point of view.

Another step which can be taken at this level, but is not covered in this study, is defining the weight factor for the departments based on the importance or criticality assigned by the top managers. In this way, the organization decides to put emphasis on some particular safety-related issues. For instance, they can define a greater weight factor for flight operation reports than maintenance or ground services reports. Of course, this step was not taken into account in this study.

Finally, by summing up the total safety risk indices of all departments and dividing them by the number of departments, the overall safety risk index of the whole organization is achieved. Again, like in the case for each department, an organization can monitor the safety trend over time and set a safety goal for it; for example, if the overall safety index of the organization is 3 , it can be defined to be lowered to 2.8 within a year.
The features of this new approach which are not present in the classical approach are: normalizing the safety index for each report, lowering the false judgment based on bias or lack of specialized technical knowledge, peer comparison and multiple prioritization of the reports, and defining the total and overall safety index both on a department and organization level. The building blocks of the model proposed here are illustrated in Figure 3 and the detailed mechanisms are explained in the next section. Particular emphasis is placed on introducing the new formula that is the starting point of risk assessment.

As mentioned before, to incorporate the effect of safety policies and organization's mission into the assessment of risk, the exponential form was chosen. Due to the nature of the Fuzzy approach, which delivers the normalized numbers of less than one, the reciprocal of the numbers derived from managing Fuzzy numbers is selected to reflect the increasing and accumulating nature of the parameters. The powers in the exponential formula come from a Fuzzy table formed to reflect the priority and preferences of the organization in weighing the risk elements.

To involve the latent impacts of safety-related accidents and incidents, impact on business was added to the formula. These changes and modifications could be introduced as:

$$
\mathrm{RI}=\text { Risk Index }=P^{1 / \alpha} S^{1 / \beta}+I^{1 / \gamma},
$$

where $P$ denotes probability, $S$ - severity, and $I$ - impact on business, and $\alpha, \beta, \gamma$ - the weighting factors for probability, severity and impact on business, respectively. The weighting coefficients need to be estimated based on pre-

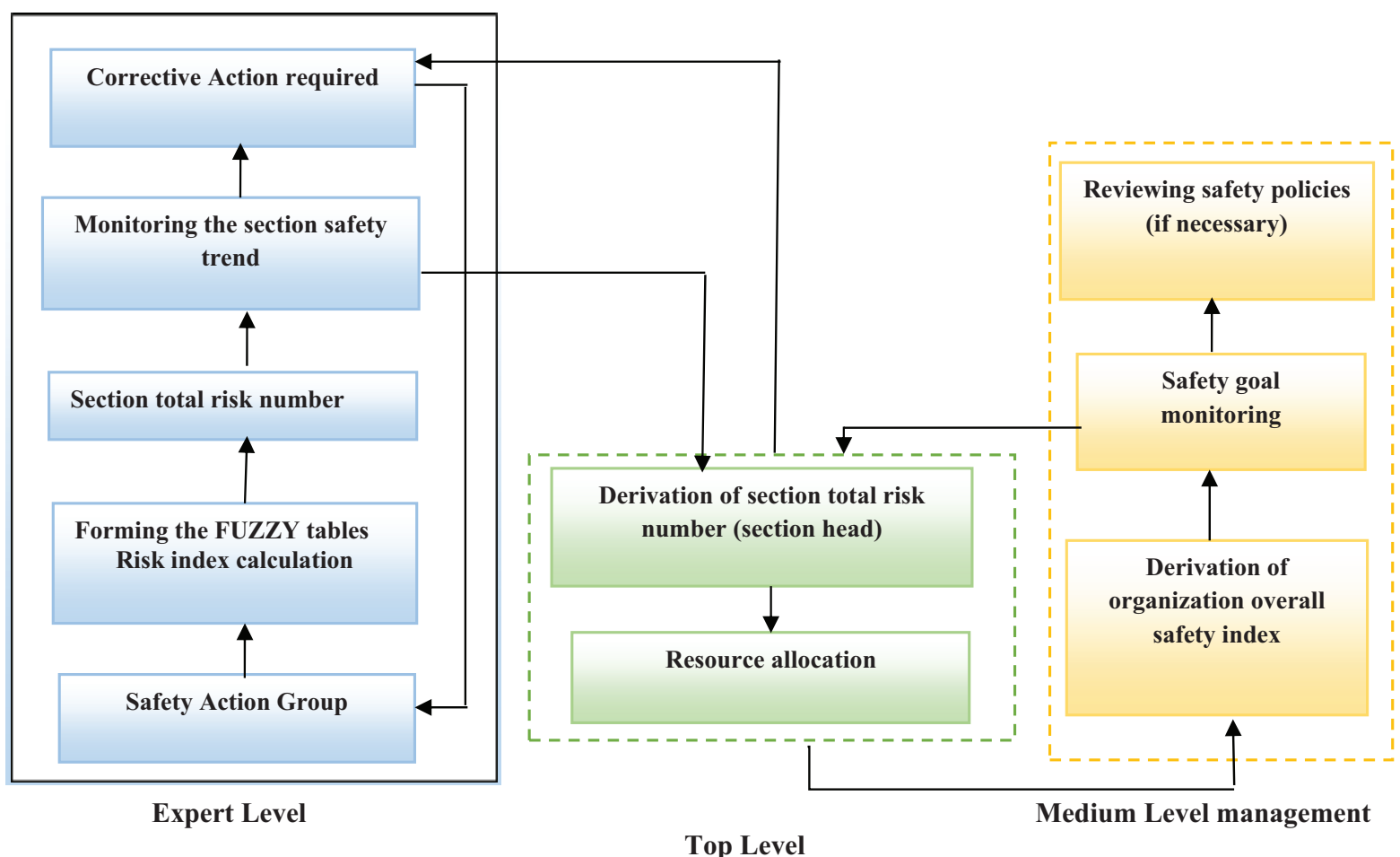

Figure 3. Building blocks (Activity assignment) of the proposed model 
vious experience, database, and an airline's priority. The methods for finding these factors and parameters will be in accordance with the Fuzzy basis described above.

\section{The field risk is formulated as:}

Field Risk $(F R)_{i}=\frac{\sum_{i=1}^{N}(R I)_{t}}{N}$,

where $R I$ is the risk index of a report and $N$ is the total number of reports received in a definite field. In this approach, the safety criteria (as a summation of risks) can be calculated for different fields, which helps to compare them and identify the vulnerable sectors.

\section{Measurement of the Overall Safety Index of the organization:}

The Field risks from different sectors: engineering and maintenance, flight operation, security, airport services, dispatch, training, air medical centers, and areas, such as ramp, hangar, etc., are summed up to obtain the Overall Safety Index of the organization:

$$
\text { Overal Risk Index }(O S I)=\sum_{i=1}^{p}\left(W_{i} \cdot F R_{i}\right),
$$

where $F R_{i}$ is the Field risk, $W_{i}$ indicates the corresponding weighing coefficients for each field derived by the same FUZZY-ANP comparison method, and $P$ is the number of operational fields (sectors/departments/areas).

\section{Results of the implementation and case study}

Figure 4 illustrates the decision tree of achieving the 3D parametrical risk assessment goal, the definition has been based on 3 factors: probability, impact on business, and severity. Four options are considered for the satisfaction and prioritizing of requirements in different fields of airline operations including aircraft maintenance, flight operations, airport services, and security. For the mutual comparison of options, measures as well as safety reports and their priority, a few experts of an Iranian airline convened and their viewpoints were gathered. To form this team, factors like sufficient knowledge for decision making and organizational familiarization were most important.

During the first step, consensus and unanimity for the establishment of criteria and options were obtained in experts' meetings. For preparing an enquiry about the importance and priority of options, criteria, factors and for prioritizing the safety reports received with respect to the three major criteria of probability, severity, and impact on business, numerous questionnaires in the forms of matrices were provided after normalization and scrutinizing. In these matrices, rows and columns correspond to criteria and reports. The figures emphasize the priority of a criterion or measure over others. To complete these matrices, experts needed to make pair comparisons on the basis of Fuzzy logic and in accordance with Fuzzy numbers in Table 1 . Then, the geometrical average was used to obtain the results (Acur \& Englyst, 2006). After the experts' ideas and their inferences in peer comparison were taken into account, the priority of criteria, options, and reports was developed. Samples of matrices completed by experts are illustrated in Tables 2, 3 and 4. The weight of each criterion, option, and safety report (Tables 2 to 4 ) was extracted according to the FANP method. Risk values for each report were calculated (Table 5). It is worth noting that

Table 2. Criteria comparison for Engineering and Maintenance Department

\begin{tabular}{|l|c|c|c|}
\hline \multicolumn{1}{|c|}{ Engineering and Maintenance Department } & Probability & Severity & Impact on business \\
\hline Probability & $(1,1,1)$ & $(0.4,0.5,0.67)$ & $(1.5,2,2.5)$ \\
\hline Severity & $(1.5,2,2.5)$ & $(1,1,1)$ & $(2.5,3,3.5)$ \\
\hline Impact on business & $(0.4,0.5,0.67)$ & $(0.29,0.33,0.4)$ & $(1,1,1)$ \\
\hline
\end{tabular}

Table 3. Criteria comparison for all departments

\begin{tabular}{|l|c|c|c|c|}
\hline \multicolumn{1}{|c|}{ Probability } & $\begin{array}{c}\text { Engineering and } \\
\text { Maintenance }\end{array}$ & $\begin{array}{c}\text { Flight Operation and } \\
\text { Dispatch }\end{array}$ & Airport Services & Security \\
\hline Engineering and Maintenance & $(1,1,1)$ & $(1,1,1)$ & $(0.4,0.5,0.67)$ & $(0.29,0.33,0.4)$ \\
\hline Flight Operation \& Dispatch & $(1,1,1)$ & $(1,1,1)$ & $(0.4,0.5,0.67)$ & $(0.29,0.33,0.4)$ \\
\hline Airport Services & $(1.5,2,2.5)$ & $(0.4,0.5,0.67)$ & $(1,1,1)$ & $(0.67,1,1.5)$ \\
\hline Security & $(2.5,3,3.5)$ & $(0.29,0.33,0.4)$ & $(0.67,1,1.5)$ & $(1,1,1)$ \\
\hline
\end{tabular}

Table 4. Criteria comparison of received reports in Airport Services Department

\begin{tabular}{|l|c|c|c|c|}
\hline \multicolumn{1}{|c|}{ Probability } & Report 1 & Report 2 & Report 3 & Report 4 \\
\hline Report 1 & $(1,1,1)$ & $(1.5,2,2.5)$ & $(2.5,3,3.5)$ & $(2.5,3,3.5)$ \\
\hline Report 2 & $(0.4,0.5,0.67)$ & $(1,1,1)$ & $(0.67,1,1.5)$ & $(0.67,1,1.5)$ \\
\hline Report 3 & $(0.29,0.33,0.4)$ & $(0.67,1,1.5)$ & $(1,1,1)$ & $(1,1,1)$ \\
\hline Report 4 & $(0.29,0.33,0.4)$ & $(1,1,1)$ & $(1,1,1)$ & $(1,1,1)$ \\
\hline
\end{tabular}


Table 5.Criteria comparison of received reports in Airport services department

\begin{tabular}{|l|c|c|c|c|}
\hline Option & Report & Report 1 & Report 2 & Report 3 \\
\hline Engineering and Maintenance & 68.88 & 5.61 & 1.0 & 4.06 \\
\hline Flight Operation and Dispatch & 81.81 & 5.56 & 8.81 & 5.99 \\
\hline Airport Services & 1.95 & 1.19 & 1.42 & 1.39 \\
\hline Security & 1.16 & 0.98 & 1.05 & 1.13 \\
\hline
\end{tabular}

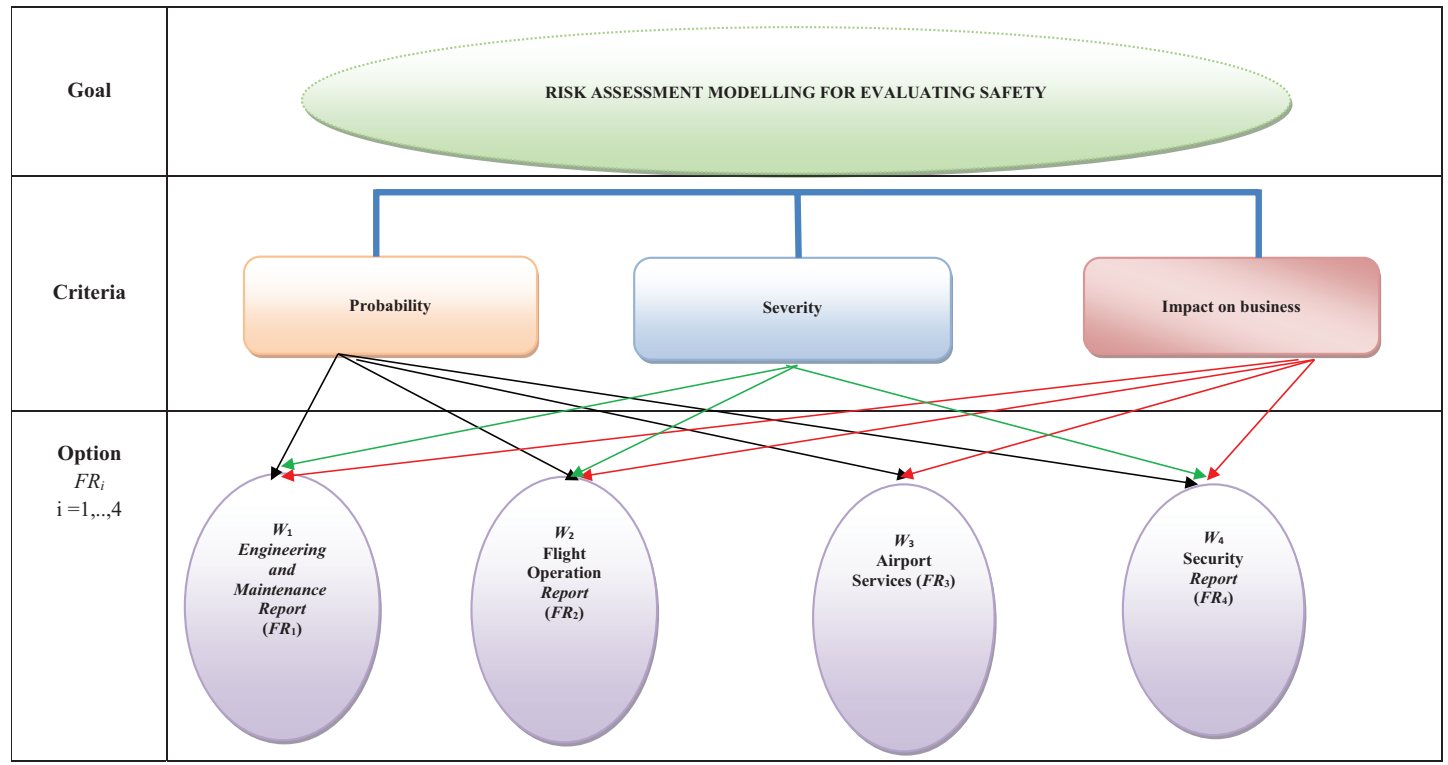

Figure 4. Goal decision tree for 3 dimensional parametric risk assessment

Table 6. Final safety index of the whole organization and departments

\begin{tabular}{|c|c|}
\hline Option Report & Safety Index \\
\hline Engineering and Maintenance & 7.289 \\
\hline Flight Operation and Dispatch & 15.665 \\
\hline Airport Services & 0.0197 \\
\hline Security & 0.011 \\
\hline Overall Safety Index & 22.98456 \\
\hline
\end{tabular}

the results for these reports have been calculated on the basis of the weights extracted using Equations (9)-(11). As mentioned above, the outputs of these calculations are presented in Table 5 (risk values of reports) and Table 6 (risk of each operation field and overall risk).

\section{Conclusions and future research}

In aviation business environment, using efficient tools for the establishment of beneficial safety policies is at the top of the agenda of all airlines. This issue can be addressed by utilizing personnel viewpoints and categorizing and prioritizing approaches for safety implementation. However, an efficient method employing realistic pair comparisons, applying subjective inferences of the organization's experts, and purging the intrinsic ambiguity of inferences is not reflected in the current safety management of airlines. To measure the safety risk using the Fuzzy hierarchical evaluation method and taking into account the experts' opinions, three criteria in four different operational fields were developed and weighed in this study. Afterwards, these weights were consolidated and the overall safety risk index was derived through "3-dimensional parametrical risk assessment". The current approach can present a model for safety risk assessment. As a result of each organization experts' viewpoints, this model is flexible and can be modified to take into account domestic and environmental parameters.

In this study, an airline has been selected as a case study. In this model, apart from the definition of a new structure for risk management, risk analysis is also restructured. Consequently, a two-dimensional classic risk formula was replaced with three-dimensional exponential formulae, which take into account "the impact on the business" as a source of risk and hazard. "The impact on business" covers all of the consequences that have originated from safety reports, but have no direct loss, damage, injury or fatality and instead have a latent or hidden impact on the reputation of the company. All the aftermath issues of the safetyrelated reports, such as delay in flight, flight cancellation, flight return, aborted takeoff, hard landing, and any other kinds of discontinuity, which may cause fear, dissatisfaction, and a negative reputation of the company, are consid- 
ered to be different examples of risks associated with "the impact on business". The strength of this new formula lies in expressing the priority of importance of the elements contributing to the risk from the management viewpoint. In view of new terms and the impact on business, it is useful to cover the latent aftermath of safety reports even if there are no accidents or incidents as risks with financial or fatal consequences. Takeoff Aborted, flight cancellation due to technical problems, hard landing provoking fear in passengers, all of which are neglected or not investigated seriously, are covered in this methodology as risk events.

Due to the differences in companies' missions, economic or political situations, lack of facilities, etc., companies may wish to put emphasis on some specific parameters contributing to safety. All organizations should have a safety goal, and, by knowing the current level of safety, could improve the level in the near future. Lack of an overall safety index for the whole organization, even if there is an index for each department, can thwart or delay the decision-making process for safety improvement.

It is proposed to develop this model by implementing other Fuzzy concepts like Fuzzy Promethee, Electre Promethee, Electre Fuzzy, and Fuzzy Topsis and consolidate them into a model related to network analysis in order to compare and select the most efficient and optimized method. It is obvious that the fundamental role in the creation of synergy and a cooperative ambience to promote safety culture in various dimensions of decision-making and management of information is played by the idea and staff initiatives to take advantage of opportunities for the identification of unique choices, which can be considered as the next steps for improving safety in an organization.

\section{Declaration of conflicting interests}

The author(s) declared no potential conflicts of interest with respect to the research, authorship, and/or publication of this article.

\section{Acknowledgements}

I would like to take this opportunity to express my profound gratitude and deep regard to dear Mr. Abdi for his valuable feedback and constant encouragement throughout the duration of writing this paper.

\section{References}

Acur, N., \& Englyst, L. (2006). Assessment of strategy formulation: how to ensure quality in process and outcome. Operation and Production Management, 26(1), 69-91. https://doi.org/10.1108/01443570610637021

Adams, E. (1976). Accident causation and the management systems. Professional Safety Journal, 21(10), 26-29.

Aven, T. (2016). Risk assessment and risk management: Review of recent advances on their fundation. European Journal of Operational Research, 235(1), 1-13.

https://doi.org/10.1016/j.ejor.2015.12.023
Bird, F. E., \& Loftus, R. G. (1976) Loss control management. Loganville, Ga: Institute Press.

Cacciabue, P. C., Cassani, M., Licata, V., Oddone, I., \& Ottomaniello, A. (2015). A practical approach to assess risk in aviation domains for safety management systems. Journal of Cognition, Technology \& Work, 17(2), 249-267.

https://doi.org/10.1007/s10111-014-0294-y

Dargi, A., Anjomshoae, A., Galankashi, M. R., Memari, A., \& Tap, M. B. M. (2014). Supplier selection: A fuzzy-ANP approach. Procedia Computer Science, 31, 691-700. https://doi.org/10.1016/j.procs.2014.05.317

Deng, F., Wang, C., \& Liang, X. (2017). Fuzzy Comprehensive Evaluation Model for Flight Safety Evaluation Research Based on an Empowerment Combination. In Proceedings of the Tenth International Conference on Management Science and Engineering Management (pp. 1479-1491). Singapore: Springer. https://doi.org/10.1007/978-981-10-1837-4_120

Di Bona, G., Silvestri, A., \& De Felice, F. (2016). An analytical model to measure the effectiveness of safety management systems: Global Safety Improve Risk Assessment (G-SIRA) method. Journal of Failure Analysis and Prevention, 16(6), 1024-1037. https://doi.org/10.1007/s11668-016-0185-Z

Ghorabaee, M. K., Amiri, M., Zavadskas, E. K., Turskis, Z., \& Antucheviciene, J. (2017). A new hybrid simulation-based assignment approach for evaluating airlines with multiple service quality criteria. Journal of Air Transport Management, 63, 45-60. https://doi.org/10.1016/j.jairtraman.2017.05.008

Griffin, T. G. C. (2010). The flight of information: New approaches for investigating aviation accident causation ( $\mathrm{PhD}$ Thesis). School of Engineering and Design, Brunel University.

Hollnagel, E., Woods, D. D., \& Leveson, N. (2007). Resilience engineering: Concepts and precepts. Aldershot, UK: Ashgate Publishing, Ltd.

International Air Transport Association. (2017). Safety report 2016 (53rd ed.). [Electtronic Version]. Retrieved from www. iata.org

International Civil Aviation Organization. (2013a). Safety Management Manual (SMM) (Doc 9859 AN/474). Retrieved from https://www.icao.int/safety/SafetyManagement/Documents/ Doc.9859.3rd\%20Edition.alltext.en.pdf

International Civil Aviation Organization. (2013b). Safety Management, Annex 19 to the Convention on International Civil Aviation. Montreal: ICAO.

Janic, M. (2000). An assessment of risk and safety in civil aviation. Journal of Air Transport Management, 6(1), 43-50. https://doi.org/10.1016/S0969-6997(99)00021-6

Johnson, W. G. (1975). MORT: The management oversight and risk tree. Journal of Safety Research, 7(1), 4-15.

Kontogiannis, T., Malakis, S., \& McDonald, N. (2017). Integrating operational and risk information with system risk models in air traffic control. Cognition, Technology \& Work, 19(2-3), 345-361. https://doi.org/10.1007/s10111-017-0409-3

Leveson, N. (2005). Risk analysis of NASA independent technical authority. Cambridge, MA: MIT press.

Leveson, N. (2011). Engineering a safer world. In Aeronautics and astronautics. Cambridge, MA, SAD: MIT press.

Lin, R. H. (2009). An integrated FANP-MOLP for supplier evaluation and order allocation. Applied Mathematical Modelling, 33(6), 2730-2736. https://doi.org/10.1016/j.apm.2008.08.021

Lind,S., Nenonen,S., \& Kivistö-Rahnasto, J. (2008). Safety risk assessment in industrial, maintenance, Journal of Quality in Maintenance Engineering, 14(2), 205-217. https://doi.org/10.1108/13552510810877692 
Maseleno, A., Hasan, M. M., Tuah, N., \& Tabbu, C. R. (2015). Fuzzy logic and mathematical theory of evidence to detect the risk of disease spreading of Highly Pathogenic Avian Influenza H5N1. Procedia Computer Science, 57, 348-357. https://doi.org/10.1016/j.procs.2015.07.349

Netjasov, F., \& Janić, M. (2008, June 1-4). A review of the research on risk and safety modelling in civil aviation. In Third International Conference on Research in Air Transportation, Fairfax, Virginia, USA (pp. 169-176). Retrieved from http:// www.icrat.org/icrat/3rd-international-conference/icrat2008_ proceedingscomplete.pdf

Noriyati, R. D., Rozaaq, W., Musyafa, A., \& Soepriyanto, A. (2015). Hazard \& operability study and determining safety integrity level on sulfur furnace unit: A case study in fertilizer industry. Procedia Manufacturing, 4, 231-236. https://doi.org/10.1016/j.promfg.2015.11.036

Oldhama, K., Stantonb, J., Bilderbecka, M., \& Spinettoa, J. (2017). Case study of a voluntary aviation safety and environmental accreditation programme. Safety Science, 96, 41-51. https://doi.org/10.1016/j.ssci.2017.02.015

Panagopoulos, I., Atkin, C. J., \& Sikora, I. (2017). Developing a performance indicators lean-sigma framework for measuring aviation system's safety performance. Transportation Research Procedia, 22, 35-44. https://doi.org/10.1016/j.trpro.2017.03.005

Perrow, C. (1999). Normal accidents: living with high-risk technologies. New Jersey: Princeton University Press.

Pillay, M. (2015). Accident causation, prevention and safety management: a review of the state-of-the-art. Procedia Manufacturing, 3, 1838-1845. https://doi.org/10.1016/j.promfg.2015.07.224

Ramkumar, M. (2016). A modified ANP and fuzzy inference system based approach for risk assessment of in-house and third party e-procurement systems. Strategic Outsourcing: An International Journal, 9(2), 159-188. https://doi.org/10.1108/SO-12-2015-0030

Ramkumar, M., Schoenherr, T., \& Jenamani, M. (2016). Risk assessment of outsourcing e-procurement services: integrating SWOT analysis with a modified ANP-based fuzzy inference system. Production Planning \& Control, 27(14), 1171-1190. https://doi.org/10.1080/09537287.2016.1190877

Rasmussen, J. (1997). Risk management in a dynamic society: a modelling problem. Safety Science, 27(2-3), 183-213. https://doi.org/10.1016/S0925-7535(97)00052-0

Reason, J. (1998). Managing the risk of organizational accidents. Burlington, USA: Ashgate Publishing Company.

Sikora, I. (2015). Risk assessment, modelling and proactive safety management system in aviation: a literature review. In Proceedings of Conference on Transportation Systems with International Participation. Retreived from http://openaccess.city. ac.uk/16320/1/Sikora_KoREMA_2015_v_2_0.pdf

Stanton, N. A. (2008). Modelling command and control. London: Taylor \& Francis Group.

Stoop, J., \& Dekker, S. (2010). Limitations of 'Swiss Cheese' models and the need for a systems approach. In Proceedings of the 41 st Annual International Seminar "Investigating ASIA in Mind-Accurate, Speedy, Independent and Authentic" (pp. 5259). Retreived from https://www.isasi.org/Documents/library/Seminar-Proceedings/Proceedings-2010.pdf

Tsay, C. Y. J., Kuo, C. C., Chao, C. J., Drury, C. G., \& Hsiao, Y. L. (2014, June). Safety culture evaluation in China Airlines: a preliminary study. In International Conference on Engineering Psychology and Cognitive Ergonomics (pp. 387-397). Cham: Springer. https://doi.org/10.1007/978-3-319-07515-0_39

Tuzkaya, U. R., \& Önüt, S. (2008). A fuzzy analytic network process based approach to transportation-mode selection between Turkey and Germany: A case study. Information Sciences, 178(15), 3133-3146. https://doi.org/10.1016/j.ins.2008.03.015

Wang, L., \& Sun, R. (2012). The development of a new safety culture evaluation index system. Procedia Engineering, 43, 331-337. https://doi.org/10.1016/j.proeng.2012.08.057

Weaver, D. (1971). Symptoms of operational error. Professional Safety Journal, 104(2), 39-42.

Wichapa, N., \& Khokhajaikiat, P. (2017). Solving multi-objective facility location problem using the fuzzy analytical hierarchy process and goal programming: a case study on infectious waste disposal centers. Operations Research Perspectives, 4, 39-48. https://doi.org/10.1016/j.orp.2017.03.002

Wise, B. (2016). Safety risk management principles from the Federal Aviation Administration. In Resident's Handbook of Medical Quality and Safety (pp. 17-20). Cham: Springer. https://doi.org/10.1007/978-3-319-24190-6_3

Xianfeng, L., \& Shengguo, H. (2012). Airport safety risk evaluation based on modification of quantitative safety management model. Procedia Engineering, 43, 238-244.

https://doi.org/10.1016/j.proeng.2012.08.041

Xueyan, S., Mingliang, Q., \& Mingang, G. (2012). Safety risk analysis in flight operations quality assurance. Systems Engineering Procedia, 5, 81-86. https://doi.org/10.1016/j.sepro.2012.04.013

Yüksel, İ., \& Dağdeviren, M. (2010). Using the fuzzy analytic network process (ANP) for Balanced Scorecard (BSC): A case study for a manufacturing firm. Expert Systems with Applications, 37(2), 1270-1278. https://doi.org/10.1016/j.eswa.2009.06.002

Zadeh, L. A. (1965). Information and control. Fuzzy Sets, 8(3), 338-353.

Zhao, X., Hwang, B. G., \& Gao, Y. (2016). A fuzzy synthetic evaluation approach for risk assessment: a case of Singapore's green projects. Journal of Cleaner Production, 115, 203-213. https://doi.org/10.1016/j.jclepro.2015.11.042

Zhao, Z., Liu, L., \& Liu, F. (2008, October). Airport internal safety risk assessment using fuzzy hierarchy process approach. In International Symposium on Computational Intelligence and Design, 2008. ISCID'08. (Vol. 1, pp. 170-173). IEEE. https://doi.org/10.1109/ISCID.2008.144

Zhou, C., \& Hu, D. (2012). Research on inducement to accident/ incident of civil aviation in southwest of china based on grey incidence analysis. Procedia Engineering, 45, 942-949. https://doi.org/10.1016/j.proeng.2012.08.263 